\title{
Transport of Lipid Vesicles by the Molecular Motor Myosin Va
}

\author{
Shane Nelson ${ }^{1}$ and Dave Warshaw ${ }^{1}$ \\ 1. Department of Molecular Physiology and Biophysics, University of Vermont, \\ Burlington VT, USA.
}

Within eukaryotic cells, vesicles and organelles are positioned and transported by molecular motor proteins that utilize cytoskeletal elements as tracks. Among the first of these motor proteins to be identified, Myosin $\mathrm{Va}$ (myoVa) moves along actin filaments and has been implicated in the transport in a wide variety of vesicles, many of which are destined for exocytosis from the cell. MyoVa has been well characterized at the single molecule level and is conceptually capable of cargo transport as a single molecule. However, experimental evidence indicates that vesicular transport is carried out by ensembles of motors [1]. There are an increasing number of studies examining the complexity of multi-motor transport. However, these studies employ synthetic cargoes such as silica beads [2] and DNA structures [3,4], which do not include one key aspect of the physiological cargoes - the fluid nature of the lipid bilayer that surrounds these structures. As the molecular motors are bound to the membrane [5], this creates a unique situation of fluid coupling between motor molecules, as well as diffusive freedom of the motor molecules over the surface of the vesicle. To explore the behavior of a lipid vesicle transported by an ensemble of molecular motors, we developed a system utilizing synthetic lipid vesicles which are coupled to recombinant myoVa. We then characterized the motion of these vesicles along purified actin filaments in an in vitro context. Using this approach, we analyzed the effects of vesicle membrane fluidity (vesicles composed of either DOPC or DPPC), vesicle diameter (varied from $100-650 \mathrm{~nm}$ ), and the surface density of myoVa motor proteins (ranging from $32-250 / \mu \mathrm{m}^{2}$ ).

Consistent with theoretical predictions [6], we observe that cargo transport by a myoVa ensemble results in a dramatic enhancement of characteristic run lengths (2-4 microns vs 0.8 microns for single motor). However, we also demonstrate that the velocity of cargo transport is not only more complex than the behavior of a single motor, but is also sensitive to aspects of the cargo. Specifically, vesicular velocity distributions show the presence of multiple populations within each experimental condition. Single myoVa molecules move along actin at $425 \pm 115 \mathrm{~nm} / \mathrm{s}$, while most vesicle preparations demonstrate a population that moves up to $30 \%$ slower than a single motor. These slow populations become slower and more predominant on gel-state vesicles and also with increasing motor density, but show no sensitivity to vesicle diameter. However, we also demonstrate the surprising presence of vesicle populations which travel at velocities exceeding those observed for a single, unloaded motor $(>700 \mathrm{~nm} / \mathrm{s})$. These usually fast velocities are unique to vesicles with fluid membranes and become even faster with increasing vesicle diameter, and slower with increasing motor density.

Mechanisms of inter-motor interference that result in reduced transport velocities by coupled motor ensembles have already been described by other groups [7]. However, the means to achieve an ensemble velocity above that of a single motor are not so 
apparent. Therefore, to explore possible mechanisms that could be responsible for the enhanced velocities seen with fluid vesicles, we developed a simple simulation of vesicular transport that utilizes kinetic and mechanical parameters of myoVa drawn from the literature. This simulation is able to recapitulate the experimental velocity distributions for each of the 14 experimental conditions that we tested. Utilizing simulated trajectories as a means to understand mechanisms underlying the experimental data, we find that the trailing motors on fluid vesicles experience an increased rate of detachment from the actin track, which leads to a forward-directed "rolling" motion of the vesicle, which generates additional forward motion.

Physiologically, cells utilize myosin Va to position and transport a variety of cargoes and organelles, yet these distinct cargoes each show unique localizations and trafficking patterns. Our findings suggest that aspects of the cargo itself may be able to regulate the behavior of the ensemble. Furthermore, these findings may bridge the gap between the reduced velocities seen in vitro and the surprisingly fast vesicular transport documented in cultured cells [8].

\section{References:}

[1] A Ashkin et al, Nature, 348 (1990), p. 346.

[2] D Jamison et al, Biophys J 99 (2010), p. 2967.

[3] N Derr et al, Science 338 (2012), p. 662.

[4] H Lu et al, J Biol Chem 287 (2012), p. 27753.

[5] J Li and A Nebenfuhr, Traffic 9 (2008), p. 290.

[6] S Klumpp and R Lipowsky, Proc Natl Acad Sci 102 (2005), p. 17284.

[7] J Driver et al, Phys Chem Chem Phys 12 (2010), p. 10398.

[8] The authors acknowledge funding from the NIH (Grant \#'s HL007944 \&

GM094229). Guy Kennedy and Samantha Previs are thanked for technical support and expertise. Dr Kathy Trybus and Elena Krementsova are thanked for providing the recombinant MyoVa HMM protein used in this study.
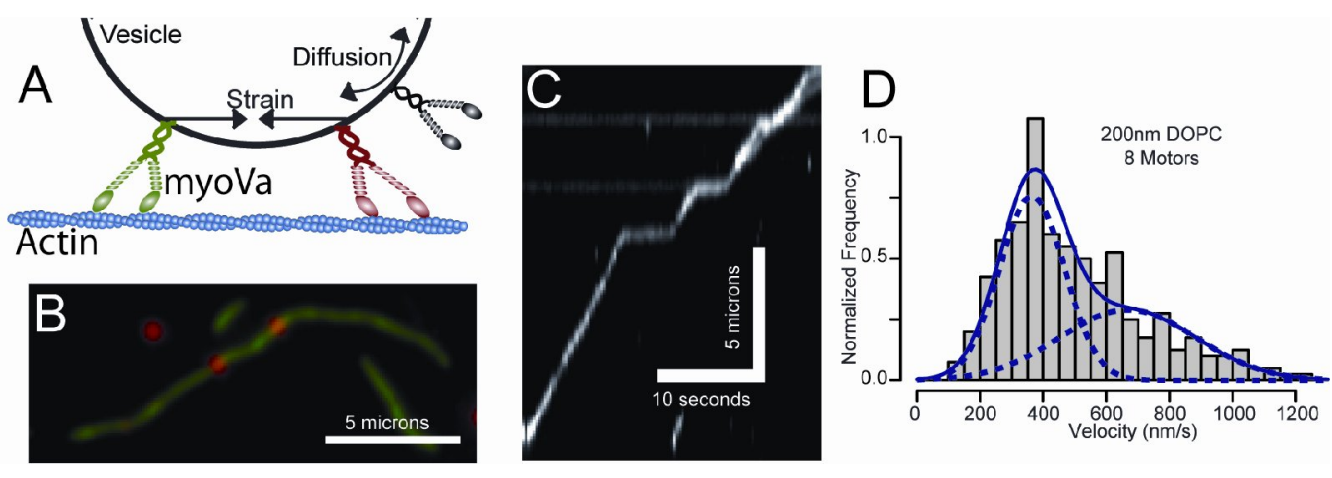

Figure 1. (A) Cartoon of Vesicular transport along actin. (B) DiI-labled vesicles (red) moving along actin (green). (C) Kymograph of vesicle movement. (D) Velocity distribution of fluid vesicle, showing presence of 2 populations (blue lines). 